\title{
Weekly Resolution Particulate Flux from a Sediment Trap in the Northern Gulf of Mexico, 2008-2012
}

By Julie N. Richey, Caitlin E. Reynolds, Eric Tappa, and Robert Thunell

Open-File Report 2014-1035

U.S. Department of the Interior

U.S. Geological Survey 


\title{
U.S. Department of the Interior SALLY JEWELL, Secretary
}

\section{U.S. Geological Survey \\ Suzette M. Kimball, Acting Director}

\author{
U.S. Geological Survey, Reston, Virginia: 2014
}

For more information on the USGS-the Federal source for science about the Earth, its natural and living resources, natural hazards, and the environment-visit http://www.usgs.gov or call 1-888-ASK-USGS

For an overview of USGS information products, including maps, imagery, and publications, visit http://www.usgs.gov/pubprod

To order this and other USGS information products, visit http://store.usgs.gov

Any use of trade, firm, or product names is for descriptive purposes only and does not imply endorsement by the U.S. Government.

Although this information product, for the most part, is in the public domain, it also may contain copyrighted materials as noted in the text. Permission to reproduce copyrighted items must be secured from the copyright owner.

Suggested citation:

Richey, J.N., Reynolds, C.E., Tappa, Eric, and Thunell, Robert, 2014, Weekly resolution particulate flux from a sediment trap in the northern Gulf of Mexico: U.S. Geological Survey Open-File Report 2014-1035, 9 p ,, http://dx.doi.org/10.3133/ofr20141035.

ISSN 2331-1258 (online) 


\section{Contents}

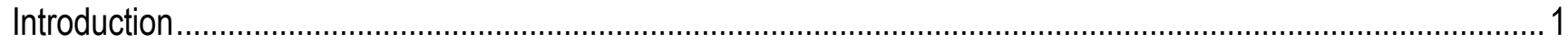

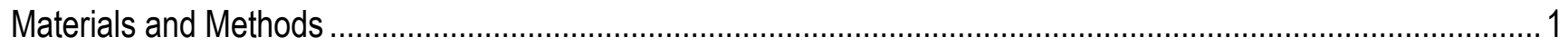

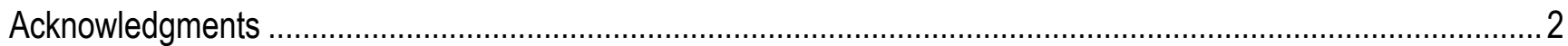

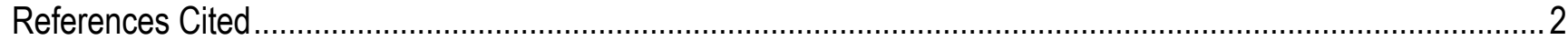

\section{Figures}

1. Average daily flux of 7- to 14-day-long sampling intervals of total mass flux and Organic Carbon (orgC) Flux for

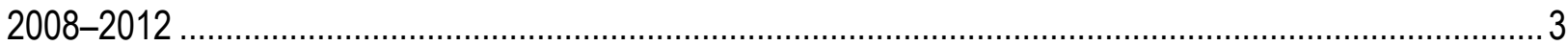

2. Average daily flux of 7-day-long sampling intervals of Calcium Carbonate $\left(\mathrm{CaCO}_{3}\right)$, Opal, Terrinegous (Terrig),

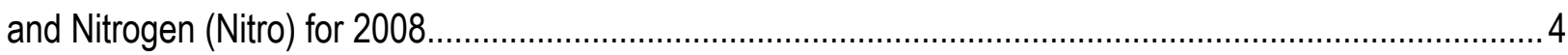

\section{Tables}

1. Average daily fluxes of total mass, Organic Carbon (orgC), Calcium Carbonate $\left(\mathrm{CaCO}_{3}\right)$, Opal, Terrinegous (Terrig), and Nitrogen (Nitro) for 2008-2012. 


\section{Conversion Factors}

\begin{tabular}{|c|c|c|}
\hline Multiply & By & To obtain \\
\hline \multicolumn{3}{|c|}{ Length } \\
\hline centimeter $(\mathrm{cm})$ & 0.394 & inch (in.) \\
\hline millimeter $(\mathrm{mm})$ & 0.039 & inch (in.) \\
\hline meter (m) & 3.281 & foot (ft) \\
\hline kilometer $(\mathrm{km})$ & 0.621 & mile (mi) \\
\hline meter $(\mathrm{m})$ & 1.094 & yard (yd) \\
\hline \multicolumn{3}{|c|}{ Area } \\
\hline square centimeter $\left(\mathrm{cm}^{2}\right)$ & 0.001 & square foot $\left(\mathrm{ft}^{2}\right)$ \\
\hline square meter $\left(\mathrm{m}^{2}\right)$ & 10.76 & square foot $\left(\mathrm{ft}^{2}\right)$ \\
\hline square centimeter $\left(\mathrm{cm}^{2}\right)$ & 0.155 & square inch $\left(\mathrm{ft}^{2}\right)$ \\
\hline \multicolumn{3}{|c|}{ Volume } \\
\hline liter $(\mathrm{L})$ & 33.82 & ounce, fluid (fl. oz) \\
\hline liter (L) & 2.113 & $\operatorname{pint}(\mathrm{pt})$ \\
\hline liter (L) & 1.057 & quart (qt) \\
\hline liter $(\mathrm{L})$ & 0.264 & gallon (gal) \\
\hline cubic meter $\left(\mathrm{m}^{3}\right)$ & 264.2 & gallon (gal) \\
\hline cubic meter $\left(\mathrm{m}^{3}\right)$ & 35.31 & cubic foot $\left(\mathrm{ft}^{3}\right)$ \\
\hline cubic meter $\left(\mathrm{m}^{3}\right)$ & 1.308 & cubic yard $\left(\mathrm{yd}^{3}\right)$ \\
\hline \multicolumn{3}{|c|}{ Mass } \\
\hline $\operatorname{gram}(\mathrm{g})$ & 0.035 & ounce, avoirdupois (oz) \\
\hline kilogram (kg) & 2.205 & pound avoirdupois (lb) \\
\hline \multicolumn{3}{|c|}{ Pressure } \\
\hline kilopascal $(\mathrm{kPa})$ & 0.010 & atmosphere, standard (atm) \\
\hline \multicolumn{3}{|c|}{ Energy } \\
\hline joule $(\mathrm{J})$ & 0.000 & kilowatthour (kWh) \\
\hline \multicolumn{3}{|c|}{ Radioactivity } \\
\hline becquerel per liter $(\mathrm{Bq} / \mathrm{L})$ & 27.027 & picocurie per liter $(\mathrm{pCi} / \mathrm{L})$ \\
\hline
\end{tabular}

Temperature in degrees Celsius $\left({ }^{\circ} \mathrm{C}\right)$ may be converted to degrees Fahrenheit $\left({ }^{\circ} \mathrm{F}\right)$ as follows:

${ }^{\circ} \mathrm{F}=\left(1.8 \mathrm{x}^{\circ} \mathrm{C}\right)+32$

Temperature in degrees Fahrenheit $\left({ }^{\circ} \mathrm{F}\right)$ may be converted to degrees Celsius $\left({ }^{\circ} \mathrm{C}\right)$ as follows:

${ }^{\circ} \mathrm{C}=\left({ }^{\circ} \mathrm{F}-32\right) / 1.8$ 


\title{
Weekly Resolution Particulate Flux from a Sediment Trap in the Northern Gulf of Mexico, 2008-2012
}

\author{
By Julie N. Richey, Caitlin E. Reynolds, Eric Tappa, and Robert Thunell
}

\section{Introduction}

The U.S. Geological Survey anchored a sediment trap in the northern Gulf of Mexico to collect time-series data on sediment flux from 2008 to 2012. There are continuous measurements of total mass flux and organic carbon flux (orgC) at 7-14 day resolution from 2008 to 2012. The flux of calcium carbonate $\left(\mathrm{CaCO}_{3}\right)$, particulate nitrogen (nitro), and biogenic silica (Opal) were also measured from January-December, 2008. The mass flux ranged from $0.01 \mathrm{~g} \mathrm{~m}^{-2} \mathrm{day}^{-1}$ (grams per square meter per day ) to $2.50 \mathrm{~g} \mathrm{~m}^{-2}$ day $^{-1}$, with a mean mass flux of $0.20 \mathrm{~g} \mathrm{~m}^{-2} \mathrm{day}^{-1}$ over the 5-year study period.

\section{Materials and Methods}

A McLane PARFLUX Mark 78 automated sediment trap was deployed in early January 2008 in approximately 1,150 meters $(\mathrm{m})$ of water depth at $27.5^{\circ} \mathrm{N}$. latitude and $90.3^{\circ} \mathrm{W}$. longitude. The trap was positioned at a depth of $700 \mathrm{~m}$ on the sediment trap mooring (Spear and Poore, 2010). The trap is equipped with 21 collection cups, all mounted on a rotating plate programmed to rotate every 7 to 14 days (representative of a 1- or 2-week collection period). Sample cups from the period of January 2008 to late May 2009 were prefilled with a buffered formalin solution made with filtered (0.44-micrometer (m) filter) seawater, with a salinity of approximently $33 \mathrm{psu}$ (practical salinity units). However, from September 2009 to the present, sample cups were prefilled with a density-gradient solution with a salinity of approximately 44 psu. Formalin (3.7 percent) and sodium borate are added to the density-gradient solution to poison, buffer and preserve the samples. The trap was recovered and redeployed every 3 months during 2008, and every 6-9 months thereafter. Sampling gaps occurred between late May 2009 and late September 2009, and between early February 2012 and late March 2012, due to scheduling problems. Nine samples from the weeks of March 17, April 7, May 5, October 22, November 19, and December 10 of 2009, and January 7, February 14 and February 24 of 2010 were not recovered due to loss of the collection cups during deployment and recovery. During visits to the trap site, water column profiles of conductivity (salinity), temperature, and depth were collected using a Sea-Bird Electronics SBE9plus (Reynolds and others, 2013). Foraminiferal assemblage composition and flux were determined from sediment trap data and published in separate reports (Reynolds and others, 2013, Poore and others, 2013).

Sediment-trap samples were wet-split into four aliquots using a precision rotary splitter at the University of South Carolina, stored in buffered, deionized water, and then refrigerated. Mass flux was determined by taking a quarter split of each cup sample, freeze drying each quarter, and then determining mass using a microbalance. All flux measurements are reported in grams per square meter per day $\left(\mathrm{g} \mathrm{m}^{-2} \mathrm{day}^{-1}\right)$. To determine organic carbon and nitrogen content, aliquots of the mass flux were rinsed with $1 \mathrm{~N}$ phosphoric acid to remove carbonates. Carbon and nitrogen content on the carbonate-free aliquots was determined via high-temperature combustion using a PerkinElmer 2400 Elemental Analyzer. Carbonate concentrations were determined with an automated acid digestion system similar to that 
described in Ostermann (1990). Biogenic Opal was quantified by the wet chemical leaching technique outlined in Mortlock and Froelich (1989). Terrigenous flux (Terrig) was calculated after accounting for Opal, $\mathrm{CaCO}_{3}$, and organic matter; the organic matter flux was extrapolated from organic carbon flux by assuming that the orgC flux accounted for 40 percent of the total organic matter.

\section{Acknowledgments}

This research was funded by the U.S. Geological Survey Climate and Land Use Research and Development program.

\section{References Cited}

Mortlock, R.A., and Froelich, P.N., 1989, A simple method for the rapid determination of biogenic opal in pelagic marine sediments: Deep-Sea Research Part A. Oceanographic Research Papers, v. 36, no. 9, p.1415-1426. [Also available at http://dx.doi.org/10.1016/0198-0149(89)90092-7.]

Ostermann, D.R., Karbott, Darrell, and Curry, W.B., 1990, Automated system to measure the carbonate concentration of sediments: Woods Hole, Mass., Woods Hole Oceanographic Institute, Technical Report WHOI-90-03. [Also available at $\underline{\text { htp: }: / d x . d o i . o r g / 10.1575 / 1912 / 998}$.]

Poore, R.Z., Tedesco, K.A., and Spear, J.W., 2013, Seasonal flux and assemblage composition of planktic foraminifers from a sediment-trap study in the northern Gulf of Mexico: Journal of Coastal Research, special issue no. 63, p. 6-19. [Also available at http://dx.doi.org/10.2112/SI63-002.1.]

Reynolds, C.E., Richey, J.N., and Poore, R.Z., 2013, Seasonal flux and assemblage composition of planktic foraminifera from the northern Gulf of Mexico, 2008-12: U.S. Geological Survey Open-File Report 2013-1243, 13 p. [Also available at http://pubs.usgs.gov/of/2013/1243/.]

Spear, J.W., and Poore, R.Z., 2010, Gulf of Mexico climate-history calibration study: U.S. Geological Survey Fact Sheet 2010-3044, 2 p. [Also available at http://pubs.usgs.gov/fs/2010/3044/.] 


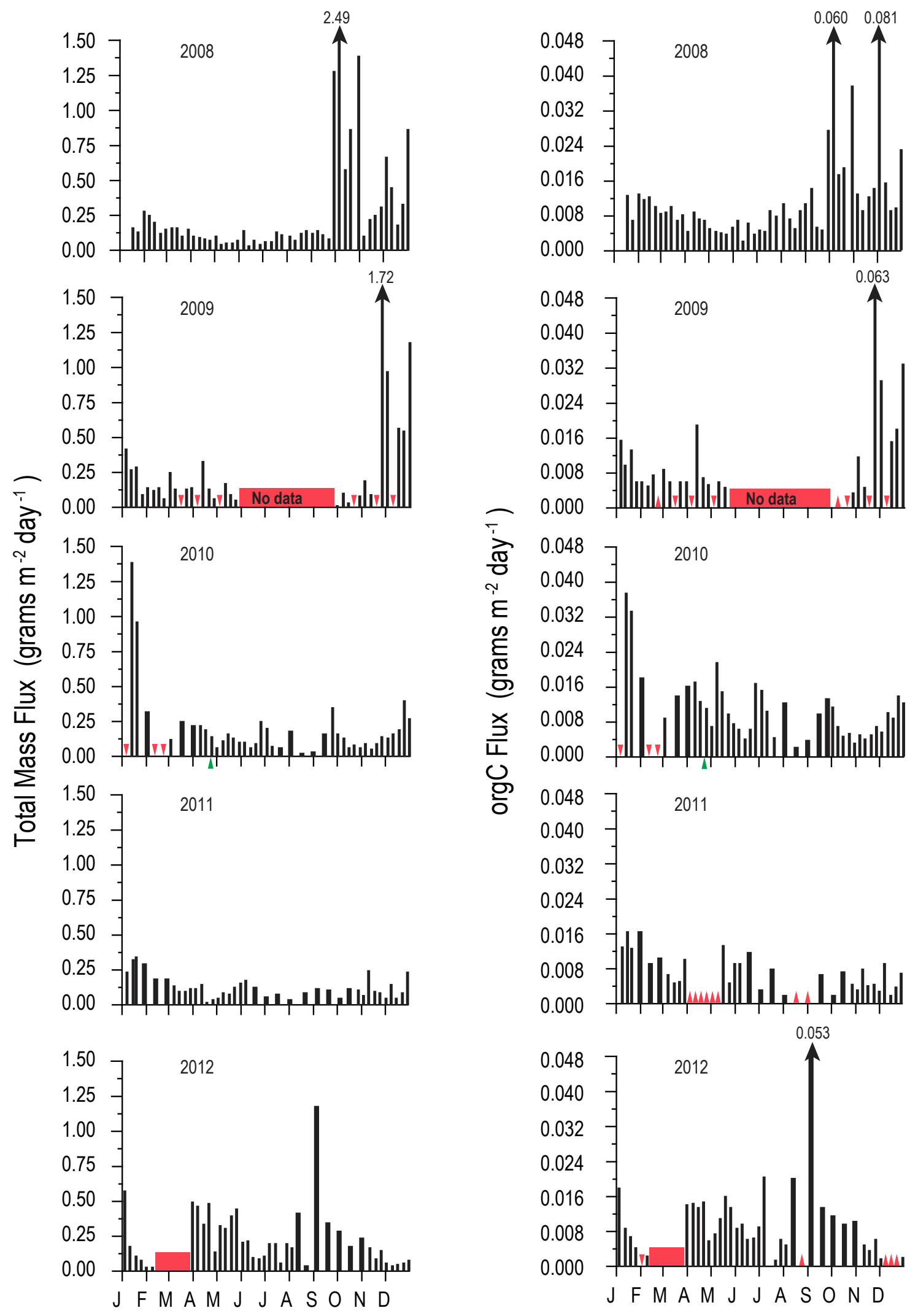

Figure 1. Average daily flux of 7- to 14-day-long sampling intervals of total mass flux and Organic Carbon (orgC) Flux for 2008-2012. Note the scale change in the y-axis. The tick marks on the $x$-axis denote the first day of each month. There was a gap in sampling (red rectangles), loss of sample cups (red inverted triangles), and limited data for processing (red triangles). Green triangle indicates Deepwater Horizon oil spill in 2010. The thick black lines represent 14 day sampling. 

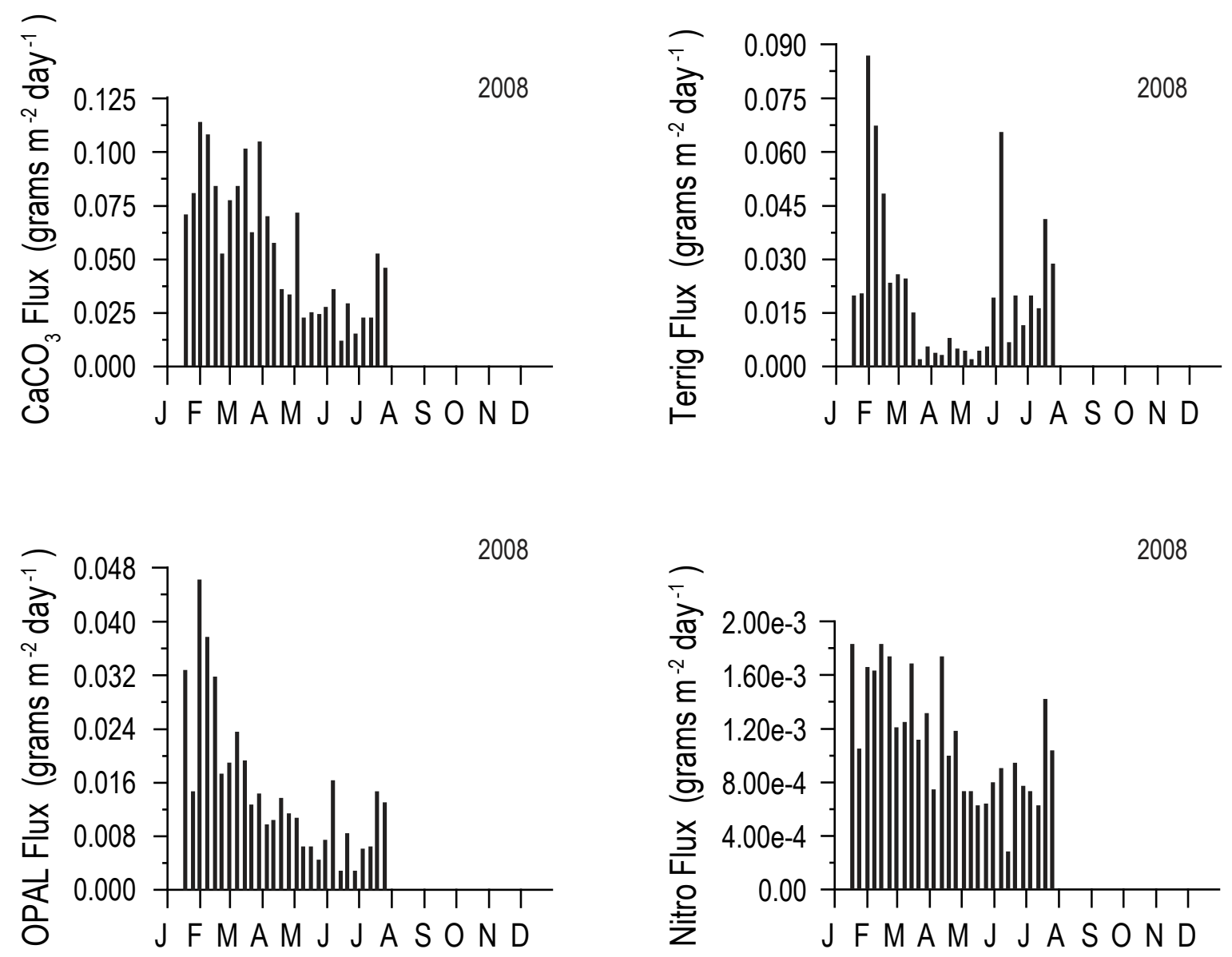

Figure 2. Average daily flux of 7-day-long sampling intervals of Calcium Carbonate $\left(\mathrm{CaCO}_{3}\right)$, Opal, Terrinegous (Terrig), and Nitrogen (Nitro) for 2008. Note the scale change in the y-axis. The tick marks on the x-axis denote the first day of each month. 
Table 1. Average daily fluxes of total mass, Organic Carbon (orgC), Calcium Carbonate $\left(\mathrm{CaCO}_{3}\right)$, Opal, Terrinegous (Terrig), and Nitrogen (Nitro) for 2008-2012.

[GMT, Gulf of Mexico Sediment Trap; OPAL, opaline]

\begin{tabular}{|c|c|c|c|c|c|c|c|c|}
\hline Sample & & Mid-Week date & $\begin{array}{l}\text { Total Mass Flux } \\
\left(\mathrm{g} / \mathrm{m}^{2} / \mathrm{day}\right)\end{array}$ & $\begin{array}{l}\text { orgC Flux } \\
\text { (gC/m²/day) }\end{array}$ & $\begin{array}{l}\mathrm{CaCO}_{3} \text { Flux } \\
\text { (g/m²/day) }\end{array}$ & $\begin{array}{l}\text { OPAL Flux } \\
\text { (g/m²/day) }\end{array}$ & $\begin{array}{l}\text { Terrig Flux } \\
\text { (g/m²/day) }\end{array}$ & $\begin{array}{l}\text { Nitro Flux } \\
\text { (g/m²/day) }\end{array}$ \\
\hline GMT-1 & 1 & 17-Jan-08 & 0.1509 & 0.0122 & 0.069 & 0.032 & 0.019 & 0.0018 \\
\hline GMT-1 & 2 & 24-Jan-08 & 0.1291 & 0.0065 & 0.079 & 0.014 & 0.020 & 0.0010 \\
\hline GMT-1 & 3 & 31-Jan-08 & 0.2760 & 0.0125 & 0.112 & 0.046 & 0.087 & 0.0016 \\
\hline GMT-1 & 4 & 7-Feb-08 & 0.2389 & 0.0113 & 0.106 & 0.037 & 0.067 & 0.0016 \\
\hline GMT-1 & 5 & $14-F e b-08$ & 0.1914 & 0.0120 & 0.082 & 0.031 & 0.048 & 0.0018 \\
\hline GMT-1 & 6 & 21-Feb-08 & 0.1146 & 0.0097 & 0.051 & 0.017 & 0.023 & 0.0017 \\
\hline GMT-1 & 7 & 28 -Feb-08 & 0.1403 & 0.0081 & 0.076 & 0.019 & 0.026 & 0.0012 \\
\hline GMT-1 & 8 & 6-Mar-08 & 0.1509 & 0.0084 & 0.082 & 0.023 & 0.024 & 0.0012 \\
\hline GMT-1 & 9 & 13-Mar-08 & 0.1571 & 0.0098 & 0.099 & 0.019 & 0.014 & 0.0017 \\
\hline GMT-1 & 10 & 20-Mar-08 & 0.0914 & 0.0066 & 0.061 & 0.012 & 0.001 & 0.0011 \\
\hline GMT-1 & 11 & 27-Mar-08 & 0.1411 & 0.0077 & 0.103 & 0.014 & 0.005 & 0.0013 \\
\hline GMT-1 & 12 & 3-Apr-08 & 0.0909 & 0.0041 & 0.068 & 0.010 & 0.003 & 0.0007 \\
\hline GMT-1 & 13 & 10-Apr-08 & 0.0891 & 0.0083 & 0.056 & 0.010 & 0.003 & 0.0017 \\
\hline GMT-1 & 14 & 17-Apr-08 & 0.0720 & 0.0069 & 0.034 & 0.013 & 0.008 & 0.0010 \\
\hline GMT-2 & 1 & 24-Apr-08 & 0.0640 & 0.0066 & 0.032 & 0.011 & 0.005 & 0.0012 \\
\hline GMT-2 & 2 & 1-May-08 & 0.0960 & 0.0046 & 0.070 & 0.010 & 0.004 & 0.0007 \\
\hline GMT-2 & 3 & 8-May-08 & 0.0389 & 0.0040 & 0.021 & 0.006 & 0.002 & 0.0007 \\
\hline GMT-2 & 4 & 15-May-08 & 0.0423 & 0.0036 & 0.023 & 0.006 & 0.004 & 0.0006 \\
\hline GMT-2 & 5 & 22-May-08 & 0.0411 & 0.0035 & 0.023 & 0.004 & 0.005 & 0.0006 \\
\hline GMT-2 & 6 & 29-May-08 & 0.0640 & 0.0049 & 0.026 & 0.007 & 0.019 & 0.0008 \\
\hline GMT-2 & 7 & 5-Jun-08 & 0.1314 & 0.0066 & 0.034 & 0.016 & 0.065 & 0.0009 \\
\hline GMT-2 & 8 & 12-Jun-08 & 0.0240 & 0.0017 & 0.011 & 0.002 & 0.006 & 0.0003 \\
\hline GMT-2 & 9 & 19-Jun-08 & 0.0697 & 0.0058 & 0.028 & 0.008 & 0.019 & 0.0009 \\
\hline GMT-2 & 10 & 26-Jun-08 & 0.0354 & 0.0033 & 0.014 & 0.002 & 0.011 & 0.0008 \\
\hline GMT-2 & 11 & 3-Jul-08 & 0.0571 & 0.0044 & 0.021 & 0.006 & 0.020 & 0.0007 \\
\hline GMT-2 & 12 & 10-Jul-08 & 0.0537 & 0.0041 & 0.021 & 0.006 & 0.016 & 0.0006 \\
\hline GMT-2 & 13 & 17-Jul-08 & 0.1280 & 0.0089 & 0.051 & 0.014 & 0.041 & 0.0014 \\
\hline GMT-2 & 14 & 24-Jul-08 & 0.1040 & 0.0074 & 0.045 & 0.013 & 0.028 & 0.0010 \\
\hline GMT-3 & 1 & 3-Aug-08 & 0.0983 & 0.0103 & & & & \\
\hline GMT-3 & 2 & 10 -Aug-08 & 0.0617 & 0.0069 & & & & \\
\hline GMT-3 & 3 & 17 -Aug-08 & 0.1166 & 0.0046 & & & & \\
\hline GMT-3 & 4 & 24-Aug-08 & 0.1360 & 0.0086 & & & & \\
\hline GMT-3 & 5 & 31-Aug-08 & 0.1177 & 0.0103 & & & & \\
\hline GMT-3 & 6 & 7-Sep-08 & 0.1360 & 0.0138 & & & & \\
\hline GMT-3 & 7 & 14-Sep-08 & 0.1086 & 0.0048 & & & & \\
\hline GMT-3 & 8 & 21-Sep-08 & 0.0754 & 0.0042 & & & & \\
\hline GMT-3 & 9 & 28-Sep-08 & 1.2709 & 0.0271 & & & & \\
\hline GMT-3 & 10 & 5-Oct-08 & 2.4937 & 0.0599 & & & & \\
\hline GMT-3 & 11 & $12-$ Oct-08 & 0.5749 & 0.0170 & & & & \\
\hline GMT-3 & 12 & 19-Oct-08 & 0.8583 & 0.0186 & & & & \\
\hline
\end{tabular}




\begin{tabular}{|c|c|c|c|c|c|c|c|c|}
\hline Sample & & Mid-Week date & $\begin{array}{l}\text { Total Mass Flux } \\
\left(\mathrm{g} / \mathrm{m}^{2} / \text { day }\right)\end{array}$ & $\begin{array}{l}\text { orgC Flux } \\
\left(\mathrm{gC} / \mathrm{m}^{2} / \text { day }\right)\end{array}$ & $\begin{array}{l}\mathrm{CaCO}_{3} \text { Flux } \\
\left(\mathrm{g} / \mathrm{m}^{2} / \text { day }\right)\end{array}$ & $\begin{array}{l}\text { OPAL Flux } \\
\text { (g/m²/day) }\end{array}$ & $\begin{array}{l}\text { Terrig Flux } \\
\text { (g/m²/day) }\end{array}$ & $\begin{array}{l}\text { Nitro Flux } \\
\text { (g/m } / \mathrm{m}^{2} / \text { day) }\end{array}$ \\
\hline GMT-4 & 1 & $29-$ Oct-08 & 1.3829 & 0.0373 & & & & \\
\hline GMT-4 & 2 & 5-Nov-08 & 0.0994 & 0.0125 & & & & \\
\hline GMT-4 & 3 & 12-Nov-08 & 0.2137 & 0.0087 & & & & \\
\hline GMT-4 & 4 & 19-Nov-08 & 0.2480 & 0.0119 & & & & \\
\hline GMT-4 & 5 & 26-Nov-08 & 0.2983 & 0.0139 & & & & \\
\hline GMT-4 & 6 & 3-Dec-08 & 0.6594 & 0.0807 & & & & \\
\hline GMT-4 & 7 & $10-$ Dec-08 & 0.4446 & 0.0152 & & & & \\
\hline GMT-4 & 8 & 17-Dec-08 & 0.1703 & 0.0086 & & & & \\
\hline GMT-4 & 9 & 24-Dec-08 & 0.3189 & 0.0094 & & & & \\
\hline GMT-4 & 10 & 31-Dec-08 & 0.8594 & 0.0228 & & & & \\
\hline GMT-4 & 11 & 6-Jan-09 & 0.4120 & 0.0152 & & & & \\
\hline GMT-5 & 1 & 12-Jan-09 & 0.2661 & 0.0093 & & & & \\
\hline GMT-5 & 2 & 19-Jan-09 & 0.2871 & 0.0128 & & & & \\
\hline GMT-5 & 3 & 26-Jan-09 & 0.0877 & 0.0056 & & & & \\
\hline GMT-5 & 4 & 2-Feb-09 & 0.1317 & 0.0054 & & & & \\
\hline GMT-5 & 5 & 9-Feb-09 & 0.1107 & 0.0045 & & & & \\
\hline GMT-5 & 6 & 16-Feb-09 & 0.1318 & 0.0071 & & & & \\
\hline GMT-5 & 7 & 23-Feb-09 & 0.0573 & & & & & \\
\hline GMT-5 & 8 & 2-Mar-09 & 0.2475 & 0.0084 & & & & \\
\hline GMT-5 & 9 & 9-Mar-09 & 0.1253 & 0.0057 & & & & \\
\hline GMT-5 & 10 & 16-Mar-09 & & & & & & \\
\hline GMT-5 & 11 & 23-Mar-09 & 0.1279 & 0.0055 & & & & \\
\hline GMT-5 & 12 & 30-Mar-09 & 0.1365 & 0.0056 & & & & \\
\hline GMT-5 & 13 & 6-Apr-09 & & & & & & \\
\hline GMT-5 & 14 & 13-Apr-09 & 0.3178 & 0.0186 & & & & \\
\hline GMT-5 & 15 & 20-Apr-09 & 0.1288 & 0.0064 & & & & \\
\hline GMT-5 & 16 & 27-Apr-09 & 0.0592 & 0.0050 & & & & \\
\hline GMT-5 & 17 & 4-Мay-09 & & & & & & \\
\hline GMT-5 & 18 & 11-May-09 & 0.1630 & 0.0057 & & & & \\
\hline GMT-5 & 19 & 18-Мay-09 & 0.0849 & 0.0042 & & & & \\
\hline GMT-5 & 20 & 24-May-09 & 0.0445 & & & & & \\
\hline GMT-6 & 1 & 30-Sep-09 & 0.0097 & & & & & \\
\hline GMT-6 & 2 & 7-Oct-09 & 0.0994 & & & & & \\
\hline GMT-6 & 3 & 14-Oct-09 & 0.0259 & & & & & \\
\hline GMT-6 & 4 & 21-Oct-09 & & & & & & \\
\hline GMT-6 & 5 & 28-Oct-09 & 0.0730 & 0.0031 & & & & \\
\hline GMT-6 & 6 & 4-Nov-09 & 0.1805 & 0.0114 & & & & \\
\hline GMT-6 & 7 & 11-Nov-09 & 0.0832 & 0.0043 & & & & \\
\hline GMT-6 & 8 & 18-Nov-09 & & & & & & \\
\hline GMT-6 & 9 & 25-Nov-09 & 1.7211 & 0.0630 & & & & \\
\hline GMT-6 & 10 & 2-Dec-09 & 0.9675 & 0.0285 & & & & \\
\hline GMT-6 & 11 & 9-Dec-09 & & & & & & \\
\hline
\end{tabular}




\begin{tabular}{|c|c|c|c|c|c|c|c|c|}
\hline Sample & & Mid-Week date & $\begin{array}{l}\text { Total Mass Flux } \\
\left(\mathrm{g} / \mathrm{m}^{2} / \text { day }\right)\end{array}$ & $\begin{array}{c}\underset{\text { orgC Flux }}{\left(\mathrm{gC} / \mathrm{m}^{2} / \text { day }\right)}\end{array}$ & $\begin{array}{l}\mathrm{CaCO}_{3} \text { Flux } \\
\left(\mathrm{g} / \mathrm{m}^{2} / \text { day }\right)\end{array}$ & $\begin{array}{l}\text { OPAL Flux } \\
\text { (g/m²/day) }\end{array}$ & $\begin{array}{l}\text { Terrig Flux } \\
\left(\mathrm{g} / \mathrm{m}^{2} / \text { day }\right)\end{array}$ & $\begin{array}{l}\text { Nitro Flux } \\
\left(\mathrm{g} / \mathrm{m}^{2} / \text { day }\right)\end{array}$ \\
\hline GMT-6 & 12 & 16-Dec-09 & 0.5563 & 0.0149 & & & & \\
\hline GMT-6 & 13 & 23-Dec-09 & 0.5449 & 0.0177 & & & & \\
\hline GMT-6 & 14 & 30-Dec-09 & 1.1707 & 0.0323 & & & & \\
\hline GMT-6 & 15 & 6-Jan-10 & & & & & & \\
\hline GMT-6 & 16 & 13-Jan-10 & 1.3810 & 0.0371 & & & & \\
\hline GMT-6 & 17 & 20-Jan-10 & 0.9555 & 0.0329 & & & & \\
\hline GMT-6 & 18 & 31-Jan-10 & 0.3169 & 0.0176 & & & & \\
\hline GMT-6 & 19 & $14-F e b-10$ & & & & & & \\
\hline GMT-6 & 20 & $24-F e b-10$ & & & & & & \\
\hline GMT-6 & 21 & 3-Mar-10 & 0.1126 & 0.0086 & & & & \\
\hline GMT-7 & 1 & 17-Mar-10 & 0.2446 & 0.0134 & & & & \\
\hline GMT-7 & 2 & 31-Mar-10 & 0.2172 & 0.0158 & & & & \\
\hline GMT-7 & 3 & 10-Apr-10 & 0.2097 & 0.0166 & & & & \\
\hline GMT-7 & 4 & 17-Apr-10 & 0.1821 & 0.0124 & & & & \\
\hline GMT-7 & 5 & 24-Apr-10 & 0.1320 & 0.0107 & & & & \\
\hline GMT-7 & 6 & 1-May-10 & 0.0530 & 0.0065 & & & & \\
\hline GMT-7 & 7 & 8-May-10 & 0.1003 & 0.0210 & & & & \\
\hline GMT-7 & 8 & 15-May-10 & 0.1502 & 0.0144 & & & & \\
\hline GMT-7 & 9 & 22-May-10 & 0.1259 & 0.0094 & & & & \\
\hline GMT-7 & 10 & 29-May-10 & 0.0959 & 0.0072 & & & & \\
\hline GMT-7 & 11 & 5-Jun-10 & 0.0920 & 0.0058 & & & & \\
\hline GMT-7 & 12 & 12-Jun-10 & 0.0558 & 0.0038 & & & & \\
\hline GMT-7 & 13 & 19-Jun-10 & 0.0851 & 0.0058 & & & & \\
\hline GMT-7 & 14 & 26-Jun-10 & 0.2481 & 0.0162 & & & & \\
\hline GMT-7 & 15 & 3-Jul-10 & 0.1902 & 0.0146 & & & & \\
\hline GMT-7 & 16 & 10-Jul-10 & 0.0690 & 0.0100 & & & & \\
\hline GMT-7 & 17 & 19-Jul-10 & 0.0512 & 0.0040 & & & & \\
\hline GMT-7 & 18 & 1-Aug-10 & 0.1767 & 0.0118 & & & & \\
\hline GMT-7 & 19 & 15-Aug-10 & 0.0162 & 0.0016 & & & & \\
\hline GMT-7 & 20 & 29-Aug-10 & 0.0225 & 0.0032 & & & & \\
\hline GMT-7 & 21 & 12 -Sep-10 & 0.1517 & 0.0095 & & & & \\
\hline GMT-8 & 1 & 24-Sep-10 & 0.3429 & 0.0127 & & & & \\
\hline GMT-8 & 2 & 1-Oct-10 & 0.1586 & 0.0109 & & & & \\
\hline GMT-8 & 3 & 8-Oct-10 & 0.1241 & 0.0064 & & & & \\
\hline GMT-8 & 4 & 15 -Oct-10 & 0.0581 & 0.0042 & & & & \\
\hline GMT-8 & 5 & $22-$ Oct-10 & 0.0801 & 0.0050 & & & & \\
\hline GMT-8 & 6 & 29-Oct-10 & 0.0534 & 0.0029 & & & & \\
\hline GMT-8 & 7 & 5-Nov-10 & 0.0895 & 0.0048 & & & & \\
\hline GMT-8 & 8 & 12-Nov-10 & 0.0502 & 0.0037 & & & & \\
\hline GMT-8 & 9 & 19-Nov-10 & 0.0840 & 0.0045 & & & & \\
\hline GMT-8 & 10 & $26-$ Nov-10 & 0.1343 & 0.0064 & & & & \\
\hline GMT-8 & 11 & 3-Dec-10 & 0.1272 & 0.0052 & & & & \\
\hline
\end{tabular}




\begin{tabular}{|c|c|c|c|c|c|c|c|c|}
\hline Sample & & Mid-Week date & $\begin{array}{l}\text { Total Mass Flux } \\
\left(\mathrm{g} / \mathrm{m}^{2} / \mathrm{day}\right)\end{array}$ & $\begin{array}{c}\text { orgC Flux } \\
\left(\mathrm{gC} / \mathrm{m}^{2} / \text { day }\right)\end{array}$ & $\begin{array}{l}\mathrm{CaCO}_{3} \text { Flux } \\
\text { (g/m²/day) }\end{array}$ & $\begin{array}{l}\text { OPAL Flux } \\
\text { (g/m²/day) }\end{array}$ & $\begin{array}{l}\text { Terrig Flux } \\
\text { (g/m²/day) }\end{array}$ & $\begin{array}{l}\text { Nitro Flux } \\
\text { (g/m } / \text { day) }\end{array}$ \\
\hline GMT-8 & 12 & 10-Dec-10 & 0.1510 & 0.0097 & & & & \\
\hline GMT-8 & 13 & 17-Dec-10 & 0.1835 & 0.0085 & & & & \\
\hline GMT-8 & 14 & 24-Dec-10 & 0.3952 & 0.0135 & & & & \\
\hline GMT-8 & 15 & 31-Dec-10 & 0.2671 & 0.0120 & & & & \\
\hline GMT-8 & 16 & 7-Jan-11 & 0.2279 & 0.0127 & & & & \\
\hline GMT-8 & 17 & 14-Jan-11 & 0.3211 & 0.0161 & & & & \\
\hline GMT-8 & 18 & 19-Jan-11 & 0.3388 & 0.0123 & & & & \\
\hline GMT-9 & 1 & 28-Jan-11 & 0.2920 & 0.0162 & & & & \\
\hline GMT-9 & 2 & $11-F e b-11$ & 0.1806 & 0.0089 & & & & \\
\hline GMT-9 & 3 & 25 -Feb-11 & 0.1800 & 0.0100 & & & & \\
\hline GMT-9 & 4 & 7-Mar-11 & 0.1326 & 0.0064 & & & & \\
\hline GMT-9 & 5 & 14-Mar-11 & 0.0857 & 0.0045 & & & & \\
\hline GMT-9 & 6 & 21-Mar-11 & 0.0949 & 0.0046 & & & & \\
\hline GMT-9 & 7 & 28-Mar-11 & 0.1074 & 0.0098 & & & & \\
\hline GMT-9 & 8 & 4-Apr-11 & 0.1051 & & & & & \\
\hline GMT-9 & 9 & 11-Apr-11 & 0.1440 & & & & & \\
\hline GMT-9 & 10 & 18-Apr-11 & 0.0069 & & & & & \\
\hline GMT-9 & 11 & 25-Apr-11 & 0.0309 & & & & & \\
\hline GMT-9 & 12 & 2-May-11 & 0.0411 & & & & & \\
\hline GMT-9 & 13 & 9-Мay-11 & 0.0789 & & & & & \\
\hline GMT-9 & 14 & 16-May-11 & 0.0731 & 0.0129 & & & & \\
\hline GMT-9 & 15 & 23-May-11 & 0.1166 & 0.0043 & & & & \\
\hline GMT-9 & 16 & 30-May-11 & 0.1486 & 0.0089 & & & & \\
\hline GMT-9 & 17 & 6-Jun-11 & 0.1680 & 0.0090 & & & & \\
\hline GMT-9 & 18 & 17-Jun-11 & 0.1211 & 0.0113 & & & & \\
\hline GMT-9 & 19 & 1-Jul-11 & 0.0486 & 0.0029 & & & & \\
\hline GMT-9 & 20 & 15-Jul-11 & 0.0680 & 0.0076 & & & & \\
\hline GMT-9 & 21 & 31-Jul-11 & 0.0289 & 0.0016 & & & & \\
\hline GMT-10 & 1 & 21-Aug-11 & 0.0771 & & & & & \\
\hline GMT-10 & 2 & 4-Sep-11 & 0.1137 & & & & & \\
\hline GMT-10 & 3 & 18-Sep-11 & 0.0966 & 0.0064 & & & & \\
\hline GMT-10 & 4 & 2-Oct-11 & 0.0360 & 0.0017 & & & & \\
\hline GMT-10 & 5 & $16-O c t-11$ & 0.1143 & 0.0069 & & & & \\
\hline GMT-10 & 6 & 26-Oct-11 & 0.1040 & 0.0042 & & & & \\
\hline GMT-10 & 7 & 2-Nov-11 & 0.0651 & 0.0028 & & & & \\
\hline GMT-10 & 8 & 9-Nov-11 & 0.2343 & 0.0076 & & & & \\
\hline GMT-10 & 9 & 16-Nov-11 & 0.0869 & 0.0038 & & & & \\
\hline GMT-10 & 10 & 23-Nov-11 & 0.0766 & 0.0041 & & & & \\
\hline GMT-10 & 11 & 30-Nov-11 & 0.0389 & 0.0024 & & & & \\
\hline GMT-10 & 12 & 7-Dec-11 & 0.1394 & 0.0088 & & & & \\
\hline GMT-10 & 13 & 14-Dec-11 & 0.0377 & 0.0016 & & & & \\
\hline GMT-10 & 14 & 21-Dec-11 & 0.0777 & 0.0034 & & & & \\
\hline
\end{tabular}




\begin{tabular}{|c|c|c|c|c|c|c|c|c|}
\hline Sample & & Mid-Week date & $\begin{array}{l}\text { Total Mass Flux } \\
\left(\mathrm{g} / \mathrm{m}^{2} / \text { day }\right)\end{array}$ & $\begin{array}{c}\underset{\operatorname{orgC} \text { Flux }}{\left(\mathrm{gC} / \mathrm{m}^{2} / \text { day }\right)}\end{array}$ & $\begin{array}{l}\mathrm{CaCO}_{3} \text { Flux } \\
\left(\mathrm{g} / \mathrm{m}^{2} / \text { day }\right)\end{array}$ & $\begin{array}{l}\text { OPAL Flux } \\
\text { (g/m²/day) }\end{array}$ & $\begin{array}{l}\text { Terrig Flux } \\
\text { (g/m²/day) }\end{array}$ & $\begin{array}{l}\text { Nitro Flux } \\
\left(\mathrm{g} / \mathrm{m}^{2} / \text { day) }\right.\end{array}$ \\
\hline GMT-10 & 15 & 28-Dec-11 & 0.2263 & 0.0068 & & & & \\
\hline GMT-10 & 16 & 4-Jan-12 & 0.5589 & 0.0177 & & & & \\
\hline GMT-10 & 17 & 11-Jan-12 & 0.1623 & 0.0085 & & & & \\
\hline GMT-10 & 18 & 18-Jan-12 & 0.0983 & 0.0064 & & & & \\
\hline GMT-10 & 19 & 25-Jan-12 & 0.0674 & 0.0040 & & & & \\
\hline GMT-10 & 20 & 1-Feb-12 & 0.0149 & & & & & \\
\hline GMT-10 & 21 & 8-Feb-12 & 0.0206 & 0.0022 & & & & \\
\hline GMT-11 & 1 & 30-Mar-12 & 0.4800 & 0.0138 & & & & \\
\hline GMT-11 & 2 & 6-Apr-12 & 0.4469 & 0.0142 & & & & \\
\hline GMT-11 & 3 & 13-Apr-12 & 0.3257 & 0.0131 & & & & \\
\hline GMT-11 & 4 & 20-Apr-12 & 0.4743 & 0.0144 & & & & \\
\hline GMT-11 & 5 & 27-Apr-12 & 0.1223 & 0.0057 & & & & \\
\hline GMT-11 & 6 & 4-May-12 & 0.3131 & 0.0073 & & & & \\
\hline GMT-11 & 7 & 11-May-12 & 0.2971 & 0.0108 & & & & \\
\hline GMT-11 & 8 & 18-May-12 & 0.3817 & 0.0157 & & & & \\
\hline GMT-11 & 9 & 25-May-12 & 0.4343 & 0.0130 & & & & \\
\hline GMT-11 & 10 & 1-Jun-12 & 0.1931 & 0.0083 & & & & \\
\hline GMT-11 & 11 & 8-Jun-12 & 0.2000 & 0.0093 & & & & \\
\hline GMT-11 & 12 & 15-Jun-12 & 0.0857 & 0.0060 & & & & \\
\hline GMT-11 & 13 & 22-Jun-12 & 0.0789 & 0.0062 & & & & \\
\hline GMT-11 & 14 & 29-Jun-12 & 0.0926 & 0.0088 & & & & \\
\hline GMT-11 & 15 & 6-Jul-12 & 0.1874 & 0.0201 & & & & \\
\hline GMT-11 & 16 & 13-Jul-12 & 0.1794 & 0.0000 & & & & \\
\hline GMT-11 & 17 & 20-Jul-12 & 0.0446 & 0.0012 & & & & \\
\hline GMT-11 & 18 & 27-Jul-12 & 0.1874 & 0.0059 & & & & \\
\hline GMT-11 & 19 & 3-Aug-12 & 0.1520 & 0.0047 & & & & \\
\hline GMT-11 & 20 & 10-Aug-12 & 0.4000 & 0.0200 & & & & \\
\hline GMT-11 & 21 & 21-Aug-12 & 0.0011 & 0.0000 & & & & \\
\hline GMT-12 & 1 & 22-Aug-12 & 0.0280 & & & & & \\
\hline GMT-12 & 2 & 5-Sep-12 & 1.1669 & 0.0531 & & & & \\
\hline GMT-12 & 3 & 19-Sep-12 & 0.3314 & 0.0130 & & & & \\
\hline GMT-12 & 4 & 3-Oct-12 & 0.2749 & 0.0112 & & & & \\
\hline GMT-12 & 5 & 17-Oct-12 & 0.1640 & 0.0095 & & & & \\
\hline GMT-12 & 6 & 31-Oct-12 & 0.2240 & 0.0099 & & & & \\
\hline GMT-12 & 7 & 10 -Nov-12 & 0.1543 & 0.0046 & & & & \\
\hline GMT-12 & 8 & 17-Nov-12 & 0.0731 & 0.0032 & & & & \\
\hline GMT-12 & 9 & 24-Nov-12 & 0.1349 & 0.0060 & & & & \\
\hline GMT-12 & 10 & 1-Dec-12 & 0.0480 & 0.0014 & & & & \\
\hline GMT-12 & 11 & 8-Dec-12 & 0.0263 & & & & & \\
\hline GMT-12 & 12 & 15-Dec-12 & 0.0377 & & & & & \\
\hline GMT-12 & 13 & 22-Dec-12 & 0.0480 & & & & & \\
\hline GMT-12 & 14 & 29-Dec-12 & 0.0697 & 0.0019 & & & & \\
\hline
\end{tabular}


